\title{
Convection in the atmospheres and envelopes of turnoff and giant branch stars of globular clusters
}

\author{
J. Montalbán ${ }^{1,2}$, F. Kupka ${ }^{3}$, F. D’Antona ${ }^{1}$, and W. Schmidt ${ }^{3,4}$ \\ 1 Osservatorio Astronomico di Roma, 00040 Monteporzio, Italy \\ 2 Instituto de Astrofísica de Canarias, 38200-La Laguna (Tenerife), Spain \\ 3 Institute for Astronomy, University of Vienna, Türkenschanzstraße 17, 1180 Wien, Austria \\ 4 Department of Physics and Astronomy, University of Wales, Cardiff, PO Box 913, Cardiff CF2 3YB, UK
}

Received 30 November 2000 / Accepted 16 February 2001

\begin{abstract}
We explore the dependence of $T_{\text {eff }}$ 's and colors of stellar models on the treatment of over-adiabatic convection, both in the atmosphere and in the interior. We compute main sequence, turnoff, and subgiant models for low metallicity stars $\left(Z=210^{-4}\right)$ using as boundary conditions two new sets of model atmospheres by the Wien group (Kupka 1996; Smalley \& Kupka 1997; Heiter et al. 2001). In these models convection is treated either in the Mixing Length Theory (MLT) or in the Full Spectrum of Turbulence (FST) formulation. We check the dependence of the $T_{\text {eff }}$ location of the HR diagram both on the optical depth $\tau$ at which the atmospheric boundary conditions are fixed, and on the convective model adopted in the atmosphere and interior. Obviously, full selfconsistency of the result is obtained only if the treatment of convection is the same in the outer layers and in the interior. We show that it is not appropriate to use MLT convection in the atmosphere and FST in the interior; if we wish to test the effect of changing the $l / H_{p}$ in the MLT, the atmospheric integration must be limited to $\tau=1$. We construct isochrones for ages of $10 \mathrm{Gyr}$ and greater, and transform the theoretical $T_{\text {eff }}$ and gravity values to the Johnson $B$ and $V$ magnitudes. The two sets of model atmospheres give small differences (up to $\sim 0.03 \mathrm{mag}$ ) for the $B-V$ color, a result of the different temperature stratification in the model atmosphere with FST convection treatment compared to that one based on MLT. The FST boundary conditions provide relative locations of turnoff and giant branch which differ from the MLT solutions, and are in better agreement with the HR diagram morphology of the Globular Cluster M 92.
\end{abstract}

Key words. stars: evolution - stars: atmospheres - convection - Galaxy: globular clusters: individual: M 92

\section{Introduction}

Nowadays, thanks to the availability of high quality observational data from both ground and space, complete color-magnitude diagrams for Globular Cluster (GC) stars begin to be available, from the upper Red Giant Branch down to the lower mass main sequence. One of the most complete examples is the cluster NGC 6397, for which there are data available down to the very end of the hydrogen burning main sequence (King et al. 1998). This poses the problem of getting reliable evolutionary information in Hubble Space Telescope colors or ground based colors for a wide range of main sequence masses and evolutionary stages. It is therefore necessary to improve the theoretical isochrones including the present day understanding of stellar physics. Furthermore, the uncertainties inherent to

Send offprint requests to: J. Montalbán, e-mail: montalbn@coma.mporzio.astro.it The isochrones are available at the WEB location http://www.mporzio.astro.it/ dantona the physical inputs in the computation must be carefully examined.

One of the most uncertain points of stellar modeling is the treatment of the outer (or less dense) layers. For the low mass main sequence models $\left(M \lesssim 0.6 M_{\odot}\right)$ the photospheric densities are large enough that convection is practically adiabatic in the layers which are most critical to determine the temperature gradients, but it is necessary to take correctly into account the detailed behaviour of the molecular opacity sources by use of full nongrey models as boundary conditions (BCs) at the surface (Baraffe et al. 1997; Cassisi et al. 2000; Montalbán et al. 2000). At larger masses $\left(M \gtrsim 0.7 M_{\odot}\right)$ convection in the atmosphere and envelope becomes overadiabatic, and the $T_{\text {eff }}$ depends on the treatment of convection more than on the atmospheric integration. For Red Giant Branch (RGB) structures, both non-grey atmospheric integration and overadiabaticity play a comparable role. So, the $T_{\text {eff }}$ location of the upper Main Sequence (MS), Turnoff (TO), and RGB stars in GCs depends on the treatment of 
overadiabatic convection, both in the interior and in the atmosphere.

In recent years, the distance scale of galactic GCs has slightly increased due to the revision of the Hipparcos subdwarf scale (Reid 1997; Gratton et al. 1997; Pont et al. 1998). As a consequence, models which were able to fit the colors of the HR diagram of GCs (including the RGB) turned out to be inadequate, and new sets of models which produce a better fit of the HR diagram morphology with the new distance scale, were proposed. The fit between theory and observations was obtained either by increasing the $\alpha$ parameter in the MLT (e.g. Cassisi et al. 1998) or by selecting color- $T_{\text {eff }}$ relations which provided better agreement (Salaris \& Weiss 1997, 1998). The problem with the RGB location when increasing the distance of GCs was also discussed (previous to Hipparcos) by Mazzitelli et al. (1995) and mainly by D'Antona et al. (1997). They adopted the Full Spectrum of Turbulence (FST) convection model by Canuto \& Mazzitelli (1991) and Canuto et al. (1996) which reproduces many observational data with no or just very moderate tuning of the local convective scale length in comparison with MLT. They showed that these models could reproduce reasonably well the relative location of MS and RGB of GCs, although the theoretical RGB remained too red by $\sim 0.02-0.04$ mag for low metallicity clusters. (Let us recall that even a few hundredths of magnitude in the color of the MS and TO are important to correctly interpret the GC HR diagrams, and that the color difference between the TO and the RGB is often used to calibrate at least relative ages of GCs - see e.g., Stetson et al. 1996 for a recent review). Those tracks were built by adopting grey atmospheric BCs, as model atmospheres computed with the FST convection model were not available. The grey atmospheric integration followed Henyey et al. (1965).

During the last few years, new and improved model atmospheres have been computed making use of the available data on atomic and molecular lines, and including approximations which try to describe the non-local character of the convective transport of energy (i.e. Kurucz 1993, 1994; Castelli et al. 1997; Bessell et al. 1998). Moreover, model atmospheres (based on the ATLAS9 code by Kurucz 1993 and 1995) which include the FST convection (Kupka 1996; Smalley \& Kupka 1997; Heiter et al. 2001) are now available. Therefore, it is now worthwhile to explore in detail the dependence of the morphology of the upper MS, TO, and RGB on the model construction.

A problem one has to consider when a model atmosphere is matched to the interior is the choice of the optical depth $\tau_{\text {match }}$ at which the atmospheric BCs are taken. From this point, the integration of atmospheric stratification supplies the values of $T_{\text {eff }}$ and $\log g$ for a given mass. If the atmospheric integration is consistent with the interior physics, the model location in the HR diagram should not depend on the choice of $\tau_{\text {match }}$ : construction of models with BCs taken at different $\tau$ 's shows how much the atmospheric model is consistent with the interior. The value of $\tau_{\text {match }}$ must be large enough for the flux to be isotropic.
On the other hand, if the boundary conditions are taken at very large values of $\tau_{\text {match }}$ the convection at this depth is almost adiabatic and the over-adiabatic part will be mostly contained in the atmospheric model. Therefore, any exploratory work on the dependence of stellar models on convection treatment must be done by building up atmospheric grids with different convection models. These calculations are time-consuming, and it is therefore customary to use a simplifying approximation: to consider the detailed atmospheric integration only down to not too large values of optical depth, and to vary the convective model in the interior. Here, we check whether this is an appropriate procedure by studying the sensitivity of the stellar model to the choice of $\tau_{\text {match }}$ and also by use of different convection models for the atmosphere and the stellar interior.

To do that we have available two new grids of model atmospheres for low metallicity $([\mathrm{M} / \mathrm{H}]=-2.0)$ stars (cf. Heiter et al. 2001). One of them describes the convective transport of energy using a MLT convection treatment with $\alpha=l / H_{p}=1.25$, and the other one includes the FST (Canuto et al. 1996) treatment. We compute full stellar models employing the ATON2.0 code (Ventura et al. 1998) which includes either MLT or FST treatment of convection in the interior, and we adopt as BCs at the surface the values of pressure and temperature from these grids, at fixed optical depth values $(\tau=1,10$, and 100).

The stars we study are in the $T_{\text {eff }}$ range between $\sim 5000 \mathrm{~K}$ and $\sim 7000 \mathrm{~K}$. At $T_{\text {eff }} \lesssim 5000 \mathrm{~K}$ triatomic molecules must be included, while at $T_{\text {eff }} \gtrsim 8000 \mathrm{~K}$ nonLTE effects begin to appear (Hauschildt et al. 1999) in the outermost layers of the photosphere. However, the regions of formation of continuum, the wings of strong lines, and most of the weak lines are situated very close to or inside the hydrogen convection zone of A stars. Moreover, for these stars the choice of a convection model (and adjustable parameters such as mixing length ratio $\left.\alpha=l / H_{\mathrm{p}}\right)$ has a very strong influence on observational quantities such as photometric colors and Balmer line profiles (Castelli et al. 1997; Smalley \& Kupka 1997; van't Veer-Menneret \& Megessier 1996; Gardiner et al. 1999). On the other hand, at $T_{\text {eff }}$ below $7000 \mathrm{~K}$ all the different convection treatments agree on the existence of a single, extensive convection zone ranging from the photosphere down to at least the region of complete ionization of helium. This makes the $T_{\text {eff }}$ range of $\sim 5000 \mathrm{~K}$ to $\sim 7000 \mathrm{~K}$ the most suitable one for our study.

In Sect. 2 we describe the main physical and chemical inputs for the different sequences of models considered in this paper. In Sect. 3 we show and analyze how the location of the models in the HR diagram is affected by the way in which convection is treated in the atmosphere and in the interior, and by the location of the interface between atmosphere and interior $\left(\tau_{\text {match }}\right)$. We see that a correct use of model atmosphere BCs requires the interior and atmospheres to be modeled with the same convection description. The effect is especially important for the FST model, which, as we show, can be correctly used only in 
conjunction with FST model atmospheres. It is not correct to match interior FST models to MLT atmospheres, unless we are dealing with models of mass low enough to have the temperature gradient very close to the adiabatic one. In the case of MLT models, the same $\alpha$ value should be used in the atmosphere and in the interior, but the differences are less important (as long as $\alpha>1$ ).

In Sect. 4 we show the results given by both sets of models (full MLT and full FST) in the observational plane. Color- $T_{\text {eff }}$ relations constructed from the two grids (MLT and FST) are used to transform the theoretical isochrones (from 10 to $18 \mathrm{Gyr}$ ) into the $B V$ Johnson system. We compare the isochrones with the color-magnitude diagram of the GC M 92 by Stetson et al. (1989). We show that the $\delta(B-V)$ between the TO and the RGB of the FST isochrones is reasonably adequate to describe it, in spite of the scarce possibility of tuning of the scale length of the FST convection model.

\section{Model inputs}

All models presented here were computed employing a helium mass fraction $Y=0.23$ and metal mass fraction $Z=210^{-4}$.

\subsection{Equation of state and opacities}

A complete description of the equation of state (EOS) of our code is given in Montalbán et al. (2000). For the present models, thermodynamics is from Rogers et al. 1996, for five different hydrogen abundances. At temperatures $T \geq 6000 \mathrm{~K}$ we adopt OPAL radiative opacities $(\bar{\kappa})$ (Roger \& Iglesias 1994, for the solar $Z$-distribution from Grevesse \& Noels 1993). In high-density $(\rho)$ regions the opacities are linearly extrapolated $(\log \bar{\kappa}$ vs. $\log \rho)$, and harmonically added to conductive opacities by Itoh \& Kohyama (1993). At lower temperatures we use Alexander \& Ferguson's (1994) molecular opacities (plus electron conduction in full ionization) for the same $\mathrm{H} / \mathrm{He}$ ratios as in the OPAL case. Opacities (and OPAL EOS) tables for $Z=210^{-4}([\mathrm{M} / \mathrm{H}]=-2.0)$ are not available in the original OPAL tables, so they have been obtained by logarithmic interpolation of $\log \bar{\kappa}$ vs. $\log \mathrm{Z}$.

\subsection{Atmospheric structure and boundary conditions}

We employ two sets of model atmospheres. These have been computed with the Kurucz (1993) ATLAS9 code, as modified by Kupka (1996) to account both for FST and MLT convection (cf. Gardiner et al. 1999; Heiter et al. 2001). We use subgrids ranging $4000 \leq T_{\text {eff }} \leq 8000 \mathrm{~K}$ and $3 \leq \log g \leq 5$, so we can build up models for masses $M \geq$ $0.5 M_{\odot}$ and luminosities $L \lesssim 10 L_{\odot}$. We match the model atmosphere with the interior at $\tau_{\text {match }}=1,10$, or 100 . Grey models are also built up for comparison, adopting the $T(\tau)$ relation by Henyey et al. (1965).

MLT model atmospheres adopt a value of scale length $\Lambda=1.25 H_{p}$. The fluxes of the FST convection models have been taken from Canuto et al. (1996). To implement them into the ATLAS9 model atmosphere code, the temperature correction algorithm, which finds $T(\tau)$ under the conditions of a constant (zero divergence) total flux, had to be modified accordingly (see Kupka 1996, expressions will be given in Heiter et al. 2001; Kupka et al. 2001).

\subsection{The scale length and the overshooting description}

The FST model not only introduces different fluxes compared to MLT, but also different prescriptions of the local scale length $\Lambda$, which have been implemented into the model atmospheres as well. For the Canuto et al. (1996) version of the FST model, we use $\Lambda=z+\alpha_{*} H_{\mathrm{p}}$, top, with $z$ as the distance to the nearest stable layer and $\alpha_{*}=0.09$ (Gardiner et al. 1999; Heiter et al. 2001, and in partic-

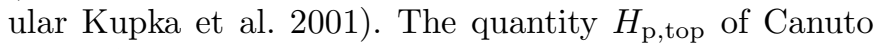
et al. (1996) is the pressure scale height at the top of the convection zone, while for the model grids used here it is the pressure scale height at the nearest stable layer, $H_{\mathrm{p} \text {,bound }}$, which may be either the top or the bottom of the convective region. This choice makes convection slightly more efficient in comparison to the original prescription of Canuto et al. (1996) and is also more consistent with the idea of allowing for a small overshoot that permits the largest scales to range beyond the convectively unstable region. In both variants this prescription is less convenient for simple downward integration schemes as used for the computation of stellar envelopes, because the size of the convection zone is not known in advance. For model atmosphere computation, there is no disadvantage because iterations on the $T(\tau)$-law have to be performed anyway. Only for model atmospheres with convection zones entirely contained in the photosphere one would expect a difference between the definition of $\Lambda$ used in the model atmospheres and the definition used by Canuto et al. (1996). For the model atmospheres used here the convection zone is large enough so that both scales lengths should be identical. Indeed, no inconsistency was found in matching the model atmospheres, based on the modified $\Lambda$, at different $\tau$ 's to stellar envelopes, based on the standard version of the Canuto et al. (1996) scale length, as we will discuss in detail below.

We note here that overshooting in the original model atmospheres of Kurucz (1993) has a very different physical idea in mind. In the Canuto et al. (1996) description, the maximum effective scale length, of an otherwise local convection model, is slightly increased to account for the slightly higher convective velocities and heat transport capabilities that can be reached in a larger convective domain. The overshooting included in Kurucz (1993) and in Castelli et al. (1997) tries to directly account for the existence of non-radiative temperature gradients in stable regions near convection zones. The procedure invented by Kurucz is to simply smooth out the convective flux over as much as $0.5 H_{\mathrm{p}}$ in each direction around the point where $\nabla=\nabla_{\mathrm{rad}}$. This accounts for the well-known 
Table 1. Sequence of computed models

\begin{tabular}{lccr}
\hline Sequence & atmosphere & interior & $\tau_{\text {match }}$ \\
\hline MLTFST1 & $\alpha=1.25$ & FST & 1 \\
MLTFST10 & $\alpha=1.25$ & FST & 10 \\
MLTFST100 & $\alpha=1.25$ & FST & 100 \\
MLT1_1.25 & $\alpha=1.25$ & MLT $\alpha=1.25$ & 1 \\
MLT10_1.25 & $\alpha=1.25$ & MLT $\alpha=1.25$ & 10 \\
MLT100_1.25 & $\alpha=1.25$ & MLT $\alpha=1.25$ & 100 \\
MLT1_1.6 & $\alpha=1.25$ & MLT $\alpha=1.6$ & 1 \\
MLT10_1.6 & $\alpha=1.25$ & MLT $\alpha=1.6$ & 10 \\
MLT100_1.6 & $\alpha=1.25$ & MLT $\alpha=1.6$ & 100 \\
FST1 & FST & FST & 1 \\
FST10 & FST & FST & 10 \\
FST100 & FST & FST & 100 \\
GMLT_1.6 & grey & MLT $\alpha=1.6$ & $2 / 3$ \\
GFST & grey & FST & $2 / 3$ \\
\hline
\end{tabular}

property found in many numerical simulations (Hurlburt et al. 1986, 1994) and in solutions of the non-local Reynolds stress equations (Kupka 1999), where $F_{\text {conv }}>0$ even if $\nabla-\nabla_{\text {ad }}<0$ in regions right next to neighboring convection zones. However, in its standard version this flux smoothing is performed in ATLAS9 over a region 510 times larger in terms of $H_{\mathrm{p}}$ than what is normally found in simulations (and also in Kupka 1999), while the adjacent, much larger region where $F_{\text {conv }}<0$ is not included in this treatment. The reason for this different extent originates from using the flux smoothing procedure as an additional degree of freedom to improve some fits of solar observations (Castelli et al. 1997). The benefits of such a procedure have been questioned by several authors, for example when discrepant results on fitting Balmer line profiles to observations were found in van't Veer-Menneret \& Megessier (1996). If a smoothing width optimized on solar observations is used to compute model atmospheres and synthetic Strömgren colors for A and F stars, the results are in clear disagreement with observations (Smalley \& Kupka 1997), while MLT based models neglecting this modification yield a much better performance. Because of these uncertainties, we have not used the overshooting prescription of Kurucz (1993) and Castelli et al. (1997) for the present study.

\subsection{The convection model in the interior}

For the interior as well, models are computed either by MLT or FST. A description of the FST model in the version used here is given in Ventura et al. (1998). We try different matches of interior and atmospheric models. Table 1 sums up the different combinations which constitute the computed sequences. The first column gives the names of the models that we shall use afterwards (MLTFST1 means model using MLT atmosphere and FST interior computation, with matching point at $\tau=1$ ). The second and the

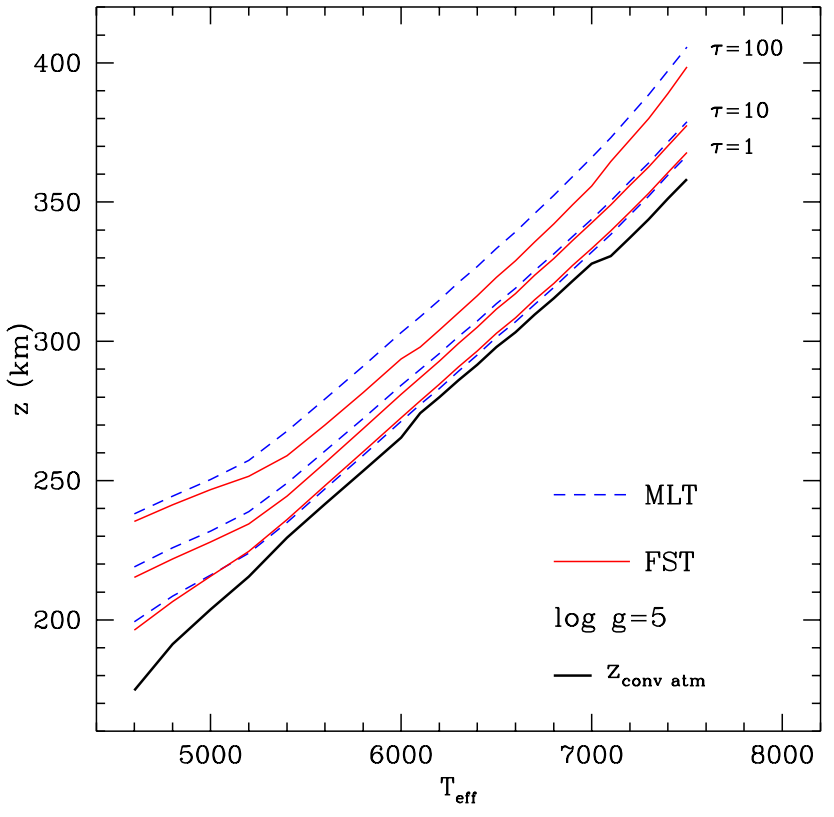

Fig. 1. Depth at which convection starts in the model atmospheres of $\log g=5$ (full heavy line) and depths, from bottom to top, of the layers $\tau=1,10$ and 100 for MLT models (dotdashed) and FST models (solid)

third columns give the characteristics of the convection model used in the atmosphere and in the interior respectively. The fourth column gives the value of the optical depth at which we perform the match between atmosphere and interior in the computation of stellar models.

An important characteristic of the FST model is that the scale length of convection is essentially the distance between the layer under examination and the top of convection. As in the model atmospheres, we define the con-

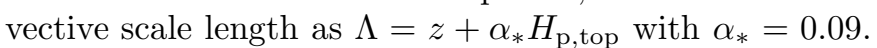
If convection starts in the atmosphere, we set $z=r+\Delta z$, where $r$ is the variable radius in the code, and $r=0$ at the atmospheric match point $\tau_{\text {match. }} \Delta z$ is the atmospheric convective depth. In Fig. 1 we show the depth in $\mathrm{km}$ of the convective boundary $\left(z_{\text {conv_atm }}\right)$ in model atmospheres of $\log g=5$ (heavy solid line). We also plot the depth $\left(z_{\tau_{\text {match }}}\right)$ of the layers corresponding to an optical depth $\tau=1,10$ and 100, for both sets of model atmospheres, MLT (dashed line) and FST (light solid line). Convection always starts at $\tau<1$. Therefore, $\Delta z=z_{\tau_{\text {match }}}-z_{\text {conv_atm }}$. We see that, whatever $\tau_{\text {match }}$, the scale length $z$ is the same.

Note also from Fig. 1 that MLT and FST model atmospheres give small differences in $z_{\tau_{\text {match }}}$, especially at $\tau_{\text {match }}=100$. This is due to the different stratification of the FST versus MLT model atmospheres.

\subsection{Comment on plane-parallel model atmospheres}

The adopted model atmospheres employ a plane-parallel (PP) approximation which is perfectly adequate for the range of $T_{\text {eff }}$ and $\log g$ considered here. In giants and 


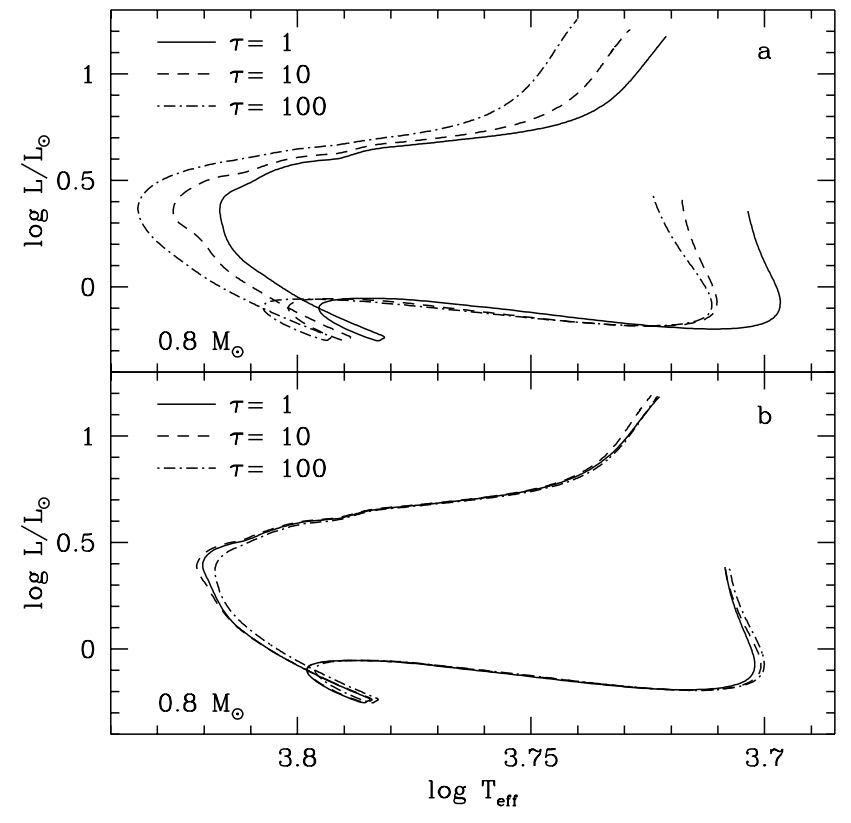

Fig. 2. Evolutionary tracks for $0.8 M_{\odot}$ computed using: a) MLT atmospheres matched with an interior computed with FST at $\tau=1,10$ and 100; and $\mathbf{b})$ FST atmospheres matched with an interior computed with FST

supergiants the atmospheric extension affects the structure of the atmospheric model, the spectra, and the colors. E.g. Plez (1990) shows that spherically symmetric (SS) models become redder when increasing the atmospheric extension at fixed $T_{\text {eff }}$ and $\log g$. The effect becomes significant below $4000 \mathrm{~K}$, and between 3000 and $4000 \mathrm{~K}$ the SS models are from 50 to $100 \mathrm{~K}$ (depending on the extension) hotter than PP models. These shifts of $T_{\text {eff }}$ have been estimated considering very low gravity models and several values of the atmospheric extension, the most extended model having $R_{\text {atm }}\left(\tau=10^{-5}\right) / R(\tau=1)=1.18$.

However, the atmosphere models used in this paper consider $T_{\text {eff }} \geq 4000 \mathrm{~K}$ and $\log g \geq 3.0$, and the atmospheric extension estimated for the models of giants computed here (which have $\left.T_{\text {eff }}>5000 \mathrm{~K}\right)$ is $R_{\text {atm }}(\tau=$ $0) / R(\tau=100) \sim 1.005$ for the RGB (and 1.001 at the TO). Therefore, for the models presented here, we can say that the effect of the sphericity is negligible compared with the other effects under exam such as the treatment of convection.

\section{Model results}

\subsection{Turn-off and giants}

First of all we show in Figs. $2 \mathrm{a}$ and $3 \mathrm{a}$ what is the result of matching the MLT atmospheres with the interior FST model. We show the evolutionary sequence of a $0.8 M_{\odot}$ star: the $T_{\text {eff }}$ of the tracks depend on $\tau_{\text {match }}$. The $\tau_{\text {match }}=1$ track is $270 \mathrm{~K}$ cooler than the $\tau_{\text {match }}=$ 100 track. This is due to the very steep temperature gradient which is characteristic of the most outer layers of the convective FST structure. If we integrate (with the

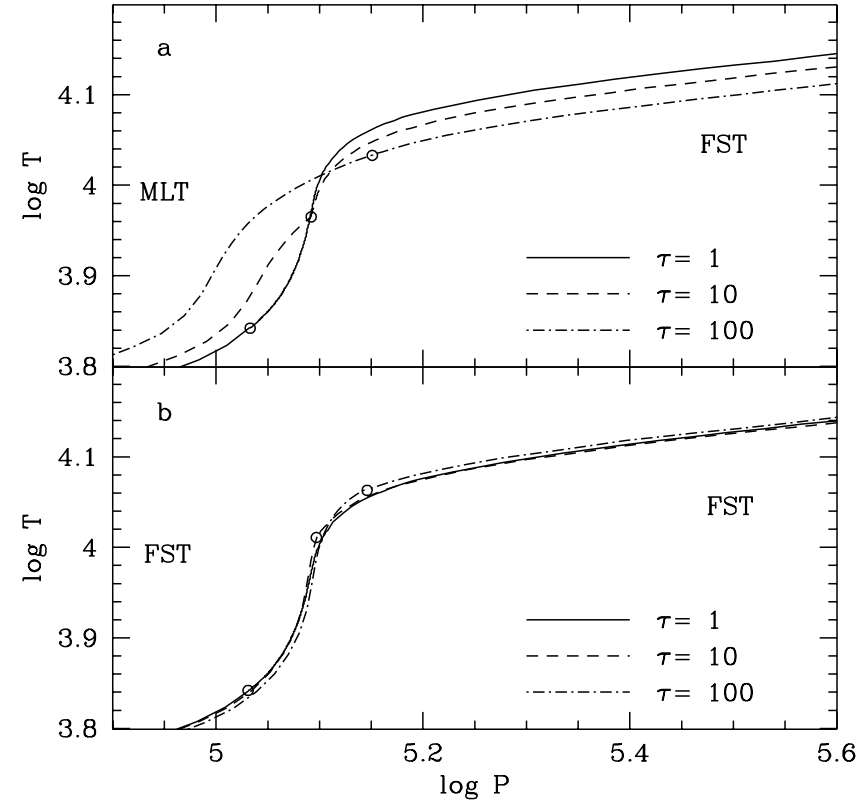

Fig. 3. The temperature stratification in the atmosphere and in the interior corresponding to the models at 10 Gyr $\left(\log L / L_{\odot} \sim 0.176\right)$ from the tracks shown in Fig. 2. Big empty dots represent the points of junction between the interior and the atmosphere

MLT) the atmosphere down to $\tau=100$, we practically get rid of the fraction of the envelope which has the steepest temperature gradient, and substitute it with the shallower gradients of the MLT atmosphere. Figure 3a shows the temperature stratification for the three different $\tau_{\text {match }}$ : it results clearly that it is not meaningful to match MLT model atmosphere to interior FST models, and that the resulting $T_{\text {eff }}$ depends very much on the incorrect mixture of convection models.

The stratification obtained by use of the FST both in the atmosphere and in the interior is, on the contrary, independent from $\tau_{\text {match }}$ as shown in Fig. $3 \mathrm{~b}$. Figure $2 \mathrm{~b}$ shows indeed that the $0.8 M_{\odot}$ full FST sequences are very similar. There remain small differences in the $T_{\text {eff }}$ of the TO, but these are not larger than $50 \mathrm{~K}$ (0.008 mag in $\left.(B-V)_{\mathrm{TO}}\right)$, and are mostly due to the fact that the TO occurs at $T_{\text {eff }}$ at which the models are very critical, as there occurs the transition from convective to radiative envelopes, producing a peculiar effect on the shape of the evolutionary tracks (see Mazzitelli et al. 1995; D'Antona et al. 1997). The RGB location also does not depend on $\tau_{\text {match }}$.

Figure 4 shows the comparison between full FST models with different BC's (grey and non-grey, upper panel) and also the comparison between full FST models and full MLT models, with the interior computed using $\alpha=1.6$ (middle panel) and $\alpha=1.25$ (lower panel). We note the known difference in the RGB between FST and MLT models (Mazzitelli et al. 1995). The maximum similarity between FST and MLT tracks is found with the $\alpha=1.6$ model. 


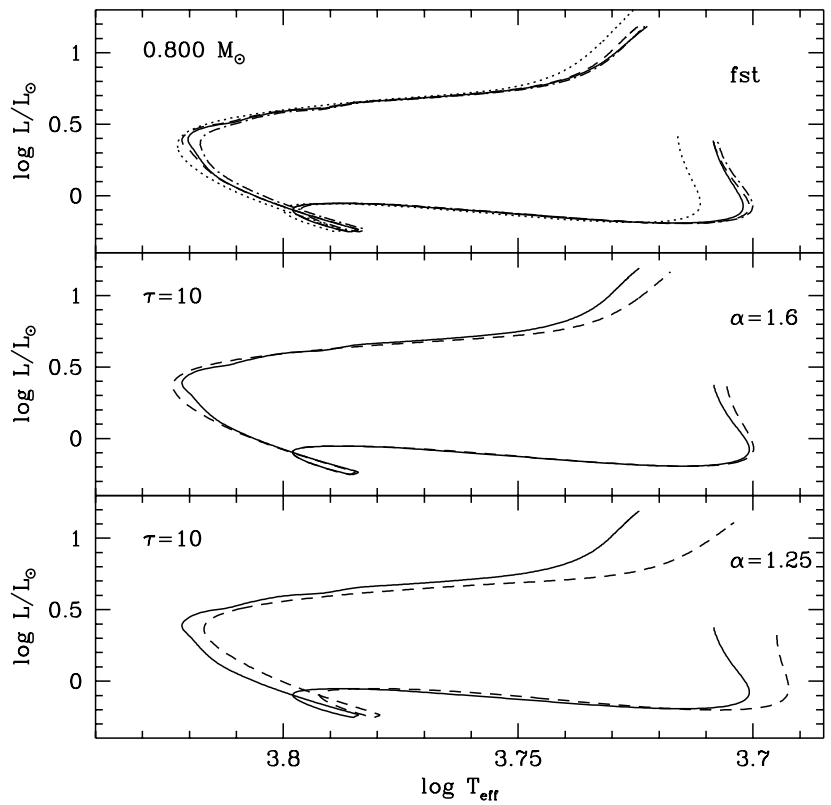

Fig. 4. Evolutionary tracks for $0.8 M_{\odot}$. Top panel: non-grey FST atmospheres matched with FST interiors at $\tau=1$ (solid line), 10 (dashed line) and 100 (dot-dashed line), and FST interior with grey atmosphere (dotted line). Middle panel: the FST $\tau=10$ track (solid line) is compared with the corresponding MLT $(\alpha=1.6)$ track (dashed line). Bottom panel: the FST $\tau=10$ track (solid line) is compared with the corresponding MLT $(\alpha=1.25)$ track (dashed line)

Figure 5 shows the full MLT models. We recall that the $\alpha=l / H_{\mathrm{p}}$ value of the model atmospheres is 1.25 . If we adopt the same $\alpha$ in the interior (top panel), we obtain models that are practically superposed in the HR diagram at the TO, and that do not appreciably differ also in the RGB (this also demonstrates that for these models any $\tau$ of integration from 1 to 100 is equivalent). If we increase the interior $\alpha$ to 1.6 (which is a value more consistent with the solar model calibration), we obtain subtle differences in the TO and RGB locations (middle panel). These differences (up to $50 \mathrm{~K}$ ) are easily understood. The larger is $\tau$, the larger is the envelope fraction in which we adopt a less efficient ( $\alpha=1.25)$ convection, the steeper is the temperature gradient and the smaller is the resulting $T_{\text {eff }}$.

Figures 4 and 5 also show the comparison with grey models. The grey FST track is $50-70 \mathrm{~K}$ hotter than the non-grey ones both at the TO and in RGB. The grey MLT tracks are identical at the TO to the non-grey tracks, but their RGBs are $\sim 80 \mathrm{~K}$ hotter $(\Delta \delta(B-V)=-0.03)$. The bottom panel of Fig. 5 shows the well known difference between the track location for the two values of $\alpha$ in MLT models. For the less efficient convection $(\alpha=1.25)$, the TO is cooler by $100 \mathrm{~K}$ and the RGB is cooler by $200 \mathrm{~K}$. From this exploration we can conclude that, in the range of $T_{\text {eff }}$ and gravities describing the TO and subgiant branch of the metal poorest GCs, MLT and FST grey atmospheric $\mathrm{BCs}$ give subtle differences with respect to non-grey BCs. If non-grey atmospheric models are not available, how-

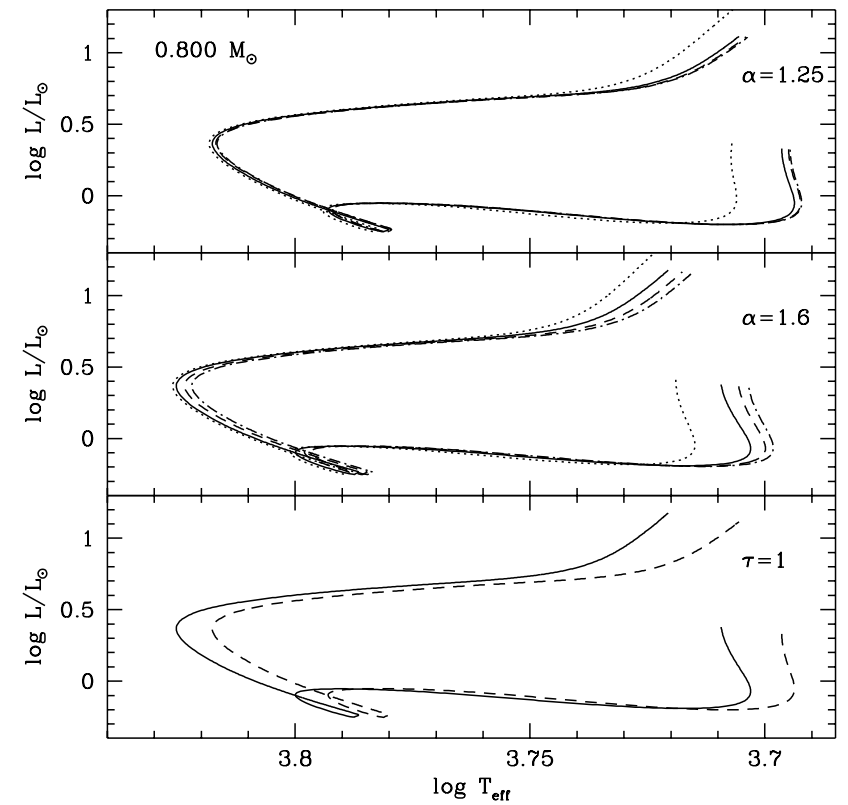

Fig. 5. Evolutionary tracks for $0.8 M_{\odot}$. Top panel: non-grey MLT $(\alpha=1.25)$ model atmospheres matched with MLT interiors $(\alpha=1.25)$ at $\tau=1$ (solid line), 10 (dashed line) and 100 (dot-dashed line). Dotted line represents the track using MLT ( $\alpha=1.25)$ internal structure and grey atmosphere. Middle panel: same as top panel using $\alpha=1.6$ for the internal structure computation. Bottom panel: comparison of $\tau=1$ tracks using $\alpha=1.25$ (dashed line) or $\alpha=1.6$ (solid line) in the interior

ever, grey models can be used, by keeping in mind that there is no difference in the TO luminosity for a given mass (and thus the ages are described correctly, as well as the mass-luminosity relation is correct), and the difference in color may amount to $0.001 \mathrm{mag}$ in MLT and $0.01 \mathrm{mag}$ in FST. However, for MLT models at least, the need of calibrating the colors on the observations, in any case, by fixing $\alpha$, leaves this problem a bit academic. For the RGB the differences are larger, and may amount to $\sim 0.04 \mathrm{mag}$, the non-grey models providing the cooler tracks.

In Table 2 we summarize these results by comparing pairs of models. We show the effects of $\tau$ and of the convection treatment on the TO location (in $T_{\text {eff }}$ and in color $(B-V))$ and on the relative quantities used to estimate GC ages, such as $\delta(B-V)$ and $\delta T_{\text {eff }}$ between the TO and the RGB (defined in all the available models as the track $T_{\text {eff }}$ at $\left.\log L / L_{\odot}=1.06\right)$.

\subsection{The main sequence}

We construct stellar models from $0.5 M_{\odot}$ to $0.9 M_{\odot}$ and from these we build up isochrones from 10 to 18 Gyr. We compare the models with the previous ones computed by Silvestri et al. (1998) with the same physical inputs apart from the atmospheric integration. The MS, TO, and RGB locations are very similar, apart from the low part of the MS, at $M<0.6 M_{\odot}$, where the present models are cooler by $\sim 150 \mathrm{~K}$. On the other hand, at masses $\leq 0.6 M_{\odot}$ the 
Table 2. Differences (MOD1-MOD2) in the TO and RGB for several pairs of models

\begin{tabular}{ll|cccc}
\hline MOD1 & MOD2 & $\Delta\left(\log T_{\text {eff }}\right)_{\text {TO }}$ & $\Delta(B-V)_{\text {TO }}$ & $\Delta\left(\delta \log T_{\text {eff }}\right)_{\text {TO-RGB }}$ & $\Delta \delta(B-V)_{\text {TO-RGB }}$ \\
\hline MLT1_1.6 & MLT100_1.6 & 0.003 & -0.008 & -0.002 & -0.02 \\
MLT1_1.25 & MLT100_1.25 & 0.001 & -0.003 & -0.001 & -0.008 \\
MLTFST1 & MLTFST100 & -0.0175 & 0.04 & 0.0035 & 0.05 \\
FST1 & FST100 & 0.003 & -0.007 & 0.0025 & 0.007 \\
MLT1_1.25 & MLT1_1.6 & -0.008 & 0.02 & 0.01 & 0.07 \\
FST10 & MLT10_1.6 & -0.002 & -0.008 & -0.01 & -0.04 \\
FST10 & MLT10_1.25 & 0.005 & -0.02 & -0.02 & -0.1 \\
GFST & GMLT_1.6 & -0.004 & 0.01 & -0.005 & -0.015 \\
GMLT_1.6 & MLT1_1.6 & 0.0008 & -0.001 & -0.006 & -0.03 \\
GFST & FST1 & 0.002 & 0.01 & -0.0035 & -0.02 \\
\hline
\end{tabular}

new models are in good agreement with those computed using Allard \& Hauschild (1997) BCs (Montalban et al. $2000)$. This confirms both that a grey $T(\tau)$ is not adequate to describe the atmosphere when molecules begin to be important, and that the present models correctly describe the MS down to at least $0.5 M_{\odot}$.

\section{Colors and comparison with observations}

In order to translate the theoretical $\log T_{\text {eff }}-\log L / L_{\odot}$ into Johnson's $B$ and $V$ bands we use the color table transformations appropriate for the two model atmosphere sets (MLT and FST). We discuss shortly the "history" of color$T_{\text {eff }}$ relations in the recent years.

\subsection{Color $-T_{\text {eff }}$ correlations}

In Fig. 6a we show the $T_{\text {eff }}-(B-V)$ correlation for $\log g=5,4$, and 3 from the Kurucz (1993) set of model atmospheres, from the set computed by Castelli, and referred as C97 by Castelli (1999), and from the present set of MLT models (MLT). The Kurucz (1993) relation, for $\log g=4$ and 3, shows a discontinuity in the colors, that was due to a problem in the original version of the approximate overshooting treatment (this anomalous behavior was first noticed by van't Veer-Menneret). The cause of the problem was later on identified by Castelli (1996) and corrected in Castelli et al. (1997). The model grids used here do not use approximate overshooting for the reasons given in Sect. 2. On the average, they give a bluer $(B-V)^{1}$. The $\mathrm{C} 97$ relation is practically equal to that one of the present models, as we should expect: the basis of the computation is the same Kurucz ATLAS9 code, with the same number of layers (72). Furthermore, microturbulence and mixing length choices are the same for the two model sets, and opacities are very similar. Figure $6 \mathrm{~b}$ shows

\footnotetext{
1 The isochrones by D'Antona et al. (1997) are very close to those we compute here in the theoretical plane, but their colors at the TO are redder by $\sim 0.06 \mathrm{mag}$, and their MS has a steeper slope: this is mainly a result of the Kurucz 1993 color transformations.
}

the comparison between the present MLT and FST color-

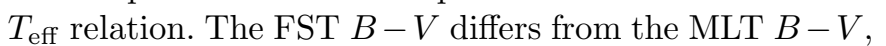
as it is to be expected due to the very different temperature stratification provided by the two convection models. Although differences are never larger than $80 \mathrm{~K}$, we must use the proper transformation for each set of models.

\subsection{Comparison with observational data}

In Fig. 7 we compare theoretical isochrones in the plane $(B-V)-M_{V}$ with the observational HR diagram of M 92 by Stetson et al. (1989). In each part of the figure we plot two pairs of isochrones, 12 and $14 \mathrm{Gyr}$, with full MLT treatment (dotted lines), and the same for full FST models (solid lines).

We show two possible comparisons: 1) We adopt a distance modulus $(m-M)_{0}=14.6$ and a reddening $E(B-V)=0.02$, the value most quoted in the literature (e.g. Buonanno et al. 1985). 2) We take a distance modulus $(m-M)_{0}=14.65$, but $E(B-V)=0.05$ (a value as large as 0.07 is suggested by King et al. 1998). Based on the Hipparcos subdwarf scale, the first distance modulus $(m-M)_{\mathrm{v}} \simeq 14.66$ is the value preferred by Pont et al. (1998), the second one $(m-M)_{\mathrm{v}} \simeq 14.8$ is preferred by Gratton et al. (1997) and Reid (1997). We see that the new FST isochrones are well consistent with the HR diagram morphology, including the RGB, for the smaller distance modulus, while the RGB is hotter by $\sim 0.05$ mag if the larger distance is chosen. Although we are aware that this result should not be over-emphasized, the use of these new FST models seems to allow a step forward in the construction of reliable models for Globular Cluster stars. MLT models would require a change in $\alpha$ from 1.6 to 2.0 at the beginning of the RGB to achieve a similar match.

The horizontal dot-dashed lines limit the MS region in which the models are less dependent both on the adopted boundary conditions and on the convection model. Nevertheless, the different $T_{\text {eff-color relations from }}$ the MLT and FST model atmospheres give a difference of $\sim 0.03 \mathrm{mag}$ in the MS location also in this range. 

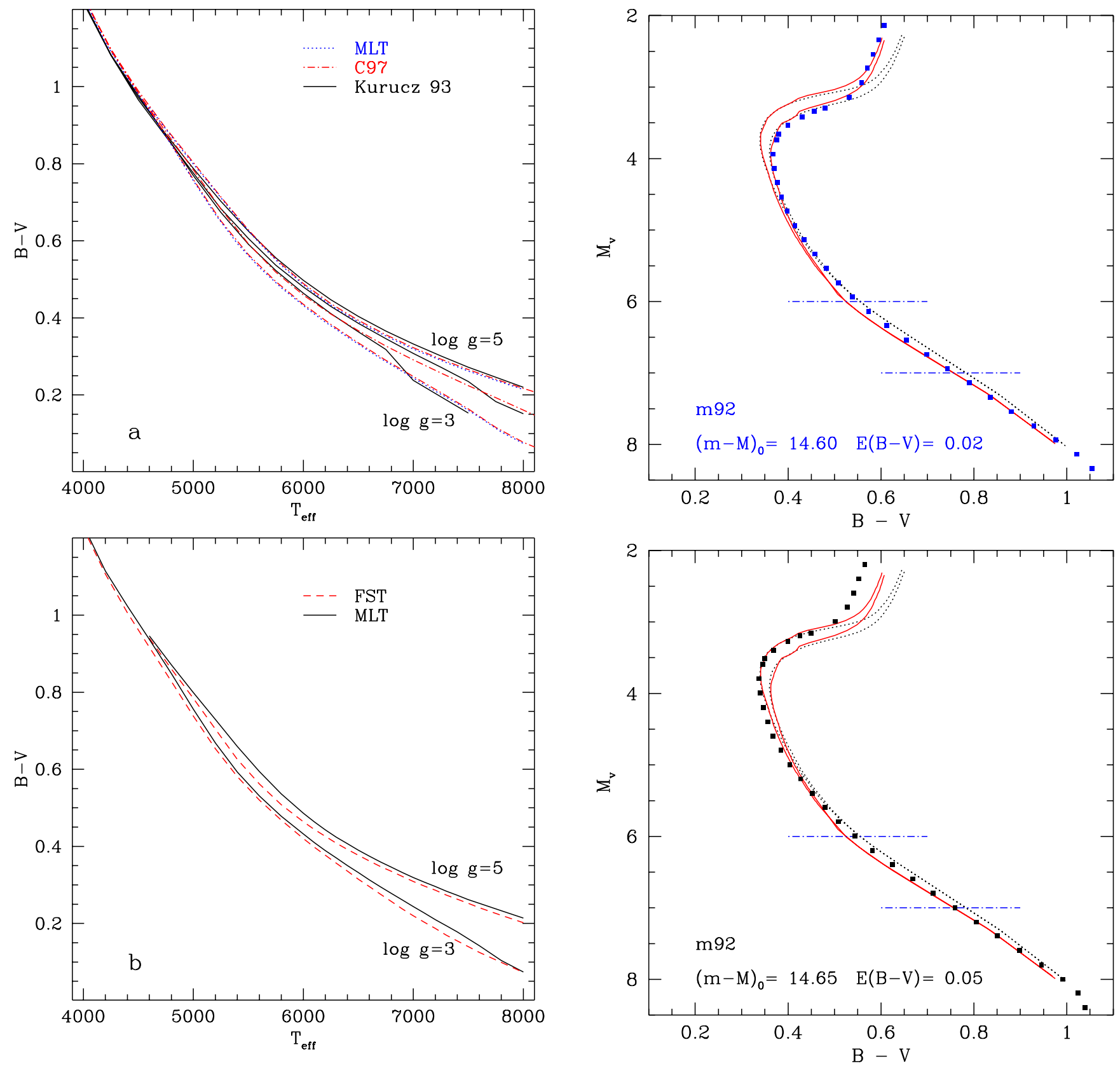

Fig. 6. The top panel shows a summary of the changes in the correlations $B-V$ versus $T_{\text {eff }}$ during recent years, due to the modification of the ATLAS9 convection routines. Three different gravities are shown ( $\log g=5,4$, and 3) for Kurucz (1993) and Castelli (1999) (C97) models, only $\log g=5$ and 3 for the present models (MLT). Our MLT colors are almost identical to the recent Castelli colors. The bottom panel shows the different $B-V$ of the FST model atmospheres with respect to MLT values

\section{Conclusions}

We computed stellar models with different combinations of interior structure (MLT, $\alpha=1.25$ and 1.6; FST) and atmospheric boundary conditions (MLT, FST, both with $\tau_{\text {match }}=1,10,100$; and grey atmosphere) in order to analyse how $T_{\text {eff }}$ and color of stellar models depend on the treatment of convection in the interior and in the atmo-

Fig. 7. The HR diagram by Stetson et al. (1989) (square points) is compared to the FST (full lines) and MLT (dotted) isochrones of 12 and $14 \mathrm{Gyr}$, by using two extreme values of reddening (and distances) from the literature (see text)

sphere. The results of this study can be summarized as follows:

- It is necessary to adopt the same convection model in the atmosphere and in the interior. This is especially true if we wish to use the FST convection since this convection treatment provides very steep gradients in the outer convective layers (see Figs. 2 and 3);

- MLT models are less sensitive to the differences between the value of $\alpha$ in the atmosphere than to the value of $\alpha$ in the interior (see Fig. 5, middle panel), at least as long as $1<\alpha<2$. To investigate the role 
of $\alpha$ for the whole models, if we have only one set of model atmospheres with a unique choice of $\alpha$, we must take the boundary conditions at small $\tau$, so that most of the overadiabatic part of the envelope is computed with the value of $\alpha$ chosen for the interior, which can be changed (Fig. 5, lower panel);

- MLT and FST grey atmospheric BCs give subtle differences with respect to non-grey BCs. There is no difference in the TO luminosity for a given mass (and thus the ages are described correctly, as well as the mass-luminosity relation), and the difference in color may amount to $0.001 \mathrm{mag}$ in MLT and $0.01 \mathrm{mag}$ in FST. However, for MLT models at least, the necessity of calibrating the colors on the observations by fixing $\alpha$, leaves this problem a bit academic. For the RGB the differences are larger, and may amount to $\sim 0.04 \mathrm{mag}$, the non-grey models providing the cooler tracks;

- A different treatment of convection in the atmospheric models (FST vs. MLT) results in small but non negligible differences (up to $\sim 0.03 \mathrm{mag}$ ) in the $T_{\text {eff }}-(B-V)$ relation (Fig. 6);

- FST models provide a good description of the TO and RGB colors for the metal poor GC M92 (Fig. 7).

We conclude that a unified model of stellar evolution including stellar atmospheres as an outer boundary condition requires a consistent formulation of convective energy transport. So, new efforts should be dedicated to develop model atmospheres with updated treatment of convection for other metallicities as well. A first step in this direction are sets of grids of model atmospheres each employing different convection models and including various metallicities from $[\mathrm{M} / \mathrm{H}]=-2.0$ to +1.0 by Heiter et al. (2001) using the ATLAS9 code. For model atmospheres also applicable to cool stars similar results are not yet available. Such new grids of model atmospheres will allow us a selfconsistent and more complete study of the HR diagram morphology of GCs.

Acknowledgements. FK and WS acknowledge support through projects S7303-AST and P13936-TEC of the Austrian Fonds zur Förderung der wissenschaftlichen Forschung, FwF.

\section{References}

Alexander, D. R., \& Ferguson, J. W . 1994, ApJ, 437, 879 Allard, F., \& Hauschildt, P. 1995, ApJ, 445, 433 (AH95)

Baraffe, I., Chabrier, G., Allard, F., \& Hauschildt, P. 1997, A\&A, 327, 1054 (BCAH97)

Caloi, V., D’Antona, F., \& Mazziteli, I. 1997, A\&A, 320, 823

Canuto, V. M., \& Mazzitelli, I. 1991, ApJ, 370, 295

Canuto, V. M., \& Mazzitelli, I. 1992, ApJ, 389, 724

Canuto, V. M., Goldman, I., \& Mazzitelli, I. 1996, ApJ, 473, 550

Cassisi, S., Castellani, V., Degl'Innocenti, S., \& Weiss, A. 1998, A\&AS, 129, 267

Cassisi, S., Castellani, V., Ciarcelluti, P., Piotto, G., \& Zoccali, M. 2000, MNRAS, 315, 679

Castelli, F. 1996, in Model Atmospheres and Spectrum Synthesis, 1996, ed. S. J. Adelman, F. Kupka, \& W. W. Weiss, ASP Conf. Ser., 108, 85
Castelli, F. 1999, A\&A, 346, 564

Castelli, F., Gratton, R. G., \& Kurucz, R. L. 1997, A\&A, 318, 841

Caughlan, G. R., \& Fowler, W. A. 1988, Atomic Data Nucl. Tab., 40, 283

Chabrier, G., \& Baraffe, I. 1997, A\&A, 327, 1039 (CB97)

D'Antona, F., Caloi, V., \& Mazzitelli, I. 1997, ApJ, 477, 519

Gardiner, R. B., Kupka, F., \& Smalley, B. 1999, A\&A, 347, 876

Gratton, R. G., Fusi Pecci, F., Carretta, E., et al. 1997, ApJ, 491, 749

Grevesse, N., \& Noels, A. 1993, in Origin and Evolution of the Elements, ed. N. Prantos, E. Vangioni-Flam, \& M. Cassé (Cambridge University Press), 14

Hauschildt, P. H., Allard, F., \& Baron, E. 1999, ApJ, 512, 377

Heiter, U., Kupka, F., van't Veer-Menneret, C., et al. 2001, $A \& A$, in prep.

Henyey, L. G., Vardya, M. S., \& Bodenheimer, P. 1965, ApJ, 142,841

Hurlburt, N. E., Toomre, J., Massaguer, J.M., \& Zahn, J.-P. 1994, ApJ, 421, 245

Hurlburt, N. E., Toomre, J., \& Massaguer, J. M. 1986, ApJ, 311,563

Itoh, N., \& Kohyama, Y. 1993, ApJ, 404, 268

Itoh, N., Mutoh, H., Hikita, A., \& Kohyama, Y. 1992, ApJ, 395,622

King, I. R., Anderson, J., Cool, A. M., \& Piotto, G. 1998, ApJ, 492, L37

King, J. R., Stephens, A., Boesgaard, A. M., \& Deliyannis, C. P. 1998, ApJ, 115, 666

Kupka, F. 1999, ApJ, 526, L45

Kupka, F., 1996, in Model Atmospheres and Spectrum Synthesis, ed. S. J. Adelman, F. Kupka, \& W. W. Weiss, ASP Conf. Ser., 108, 73

Kupka, F., Heiter, U., van't Veer-Menneret, C., et al. 2001, A\&A, in prep.

Kurucz, R. L. 1995, CD-ROM, No. 13, revised

Kurucz, R. L. 1993, ATLAS9 Stellar Atmosphere Programs and $2 \mathrm{~km} \mathrm{~s}^{-1}$ grid (Kurucz CD-ROM, No. 13)

Mazzitelli, I., D'Antona, F., \& Caloi, V. 1995, A\&A, 302, 382

Montalbán, J., D'Antona, F., \& Mazzitelli, I. 2000, A\&A, 360, 935

Plez, B. 1990, Mem. S. A. It., 61, No. 3, 765

Pont, F., Mayor, M., Turon, C., \& Vandenberg, D. A. 1998, A\&A, 329, 87

Reid I. N. 1997, AJ, 114, 161

Rogers, F. J., Swenson, F. J., \& Iglesias, C. A. 1996, ApJ, 456, 902

Salaris, A., \& Weiss, A. 1997, A\&A, 327, 107

Salaris, A., \& Weiss, A. 1998, A\&A, 335, 943

Saumon, D., Chabrier, G., \& van Horn, H. M. 1995, ApJS, 99, $713(\mathrm{SCVH})$

Saumon, D., Bergeron, P., Lunine, J. I., Hubbard, W. B., \& Burrows, A. 1994, ApJ, 424, 333

Silvestri, F., Ventura, P., D’Antona, F., \& Mazzitelli, I. 1998 ApJ, 509, 192

Smalley, B., \& Kupka, F. 1997, A\&A, 328, 349

Stetson, P. B., VandenBerg, D. A., \& Bolte, M. 1996, PASP, 108,560

van't Veer-Menneret, C., \& Megessier, C. 1996, A\&A, 309, 879

Ventura, P., Zeppieri, A., Mazzitelli, I., \& D'Antona, F. 1998, A\&A, 334, 953 